\title{
Code Aided Joint Frame Synchronization and Channel Estimation for Uplink MC-CDMA in the Presence of Narrowband Interference
}

\author{
Mohamed Marey ${ }^{1}$, Mamoun Guenach ${ }^{2}$ and Heidi Steendam ${ }^{1}$ \\ ${ }^{1}$ DIGCOM research group, TELIN Dept., Ghent University \\ Sint-Pietersnieuwstraat 41, 9000 Gent, Belgium \\ E-mail: \{mohamed, hs\}@telin.ugent.be \\ ${ }^{2}$ Alcatel-Lucent Bell Labs Antwerp, Belgium \\ E-mail: guenach@ieee.org
}

\section{ABSTRACT}

Spectrum-overlay scenarios for wide-band multi-carrier (MC) systems bring new technical challenges along that must be considered during the system design. In such scenarios, the receiver has to perform actions, such as channel estimation and synchronization, in the presence of possibly strong inband interference. In this paper, we investigate the impact of digitally modulated narrowband interference (NBI) on the iterative algorithm for joint channel estimation and frame synchronization as proposed in [1] for uplink MC-CDMA. Moreover, we modify the algorithm to have better performance in the presence of NBI. The performance of the modified algorithm taking into account the statistics of NBI is verified through computer simulations and compared with the results from [1].

\section{INTRODUCTION}

Multi-carrier code division multiple access (MC-CDMA), which is based on a combination of CDMA and orthogonal frequency division multiplexing (OFDM), has recently received increasing interest for high data rate transmission [2], [3]. The scarcity of available bandwidth typically necessitates spectrum sharing between legacy and new MC-CDMA systems [4]. These legacy systems are considered as narrowband interference (NBI), hampering the proper action of the MCCDMA system. In this paper, we adapt the iterative maximum likelihood joint frame synchronization and channel estimation algorithm for uplink MC-CDMA presented in [1] to have better performance in the presence of NBI.

The rest of the paper is organized as follows. In section II, a MC-CDMA system model and a narrow-band interference model are described. The derivation of the modified algorithm in the presence in NBI is illustrated in section III. Simulation results are shown in section IV. Finally, the conclusions are given in section $\mathrm{V}$.

\section{SYSTEM DESCRIPTION}

\section{A. Asynchronous Uplink MC-CDMA System Model}

In this section, we summarize the system model proposed in [1]. We consider an uplink MC-CDMA system with $K_{u}$ users. Each active user transmits frames consisting of $M \mathrm{MC}$ blocks to the base station. One frame belonging to the $k$ th user is generated as follows (see Fig. 1): a block of $N_{b}$ information bits is encoded, resulting in $N_{c}$ coded bits. After interleaving, these $N_{c}$ coded bits are mapped onto a sequence of $N_{d}$ symbols, belonging to a unit-energy $2^{q}$-point constellation $\Omega$ (with $N_{d}=N_{c} / q$ ). After insertion of $N_{p}$ pilot symbols, we obtain the vector $\mathbf{d}^{(k)}=\left[d_{0}^{(k)}, \ldots, d_{N_{d}+N_{p}-1}^{(k)}\right]^{T}$. The resulting $N_{d}+N_{p}$ symbols are broken into $M$ blocks of length $P$, where $P=\left(N_{d}+N_{p}\right) / M$. Further, the blocks of length $P$ are spread by using a spreading code of length $N_{s}$, serial-toparallel (S/P) converted, interleaved in the frequency domain, converted to the time domain using an $N$-point inverse Fourier transform (IFFT), where $N \geq P \cdot N_{s}$, and finally the cyclic prefix of $\nu$ samples is inserted. We can write the $n$-th timedomain sample $(n=-\nu, \ldots, N-1)$ in the $j$-th MC block of the $k$-th user as

$$
s_{j, n}^{(k)}=\sqrt{\frac{E_{s}}{N+\nu}} \sum_{p=0}^{P-1} \sum_{s=0}^{N_{s}-1} d_{j, p}^{(k)} c_{s}^{(k)} e^{j 2 \pi m_{p, s}^{(k)} n / N}
$$

where $d_{j, p}^{(k)}$ is the $p$-th modulated data symbol in the $j$-th MCCDMA block of the $k$-th user, $c_{s}^{(k)}$ is the $s$-th chip of the spreading code of length $N_{s}$ for the $k$-th user, $E_{s}$ denotes the energy per symbol, and $m_{p, s}^{(k)}$ is the index of the modulated sub-carrier. We further define the MC block duration $T$ and the sampling duration $T_{o}=T / N_{T}$, where $N_{T}=N+\nu$. The signal of each user is shaped with a normalized transmit filter and transmitted over the channel to the base stations.

The base station has to process $M$ MC blocks for each of the $K_{u}$ users. As shown in [1], the received sequence

$$
\mathbf{r}=\left[r\left(-\nu T_{o}\right), \ldots, r\left(M T+\left(\Delta_{\max }+L-\nu-2\right) T_{o}\right)\right]^{T}
$$

contains sufficient information to estimate the delay shift $\Delta^{(k)}$ and the channel impulse response (CIR) $\mathbf{h}^{(k)}$ for each user, and to detect the data, where $\Delta_{\max }$ denotes to the maximum integer part of the propagation delay.

To recover the information of user $k$, the contributions of other users must be removed from the received signal $\mathbf{r}$. This process is known as time-domain multiple access interference cancellation (TD-MAIC) [1]. After that, we perform a single user detection in the frequency domain. The TD-signals of the different users can be reconstructed by using the soft information from the decoders and we can again perform TD-MAIC, and so forth. At the first iteration, no TD-MAIC is performed, so we must rely on the spreading to obtain reasonable bit error rate (BER) performance. 


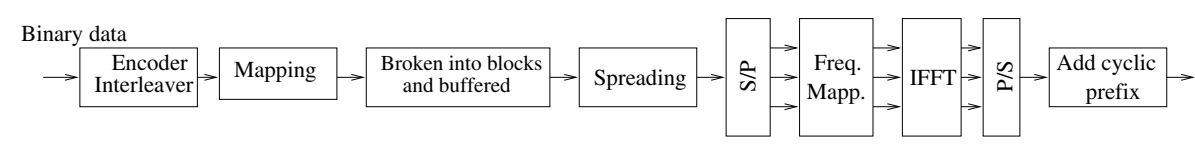

Fig. 1. Transmitter for one user.

\section{B. Narrowband Interference Model}

We assume the NBI consists of $N_{I}$ digital modulated signals. Following the interference model from [5], the total NBI signal at the output of the matched filter of the MC receiver may be written as

$$
r_{I}(t)=\sum_{l=1}^{N_{I}} \sum_{i=-\infty}^{\infty} z_{i, l} e^{j 2 \pi f_{c, l} i T_{l}} g_{l}\left(t-i T_{l}\right)
$$

where $f_{c, l}$ is the carrier frequency deviation from the MC central carrier frequency $f_{0}$ of the $l$ th interferer, $z_{h, l}$ is the $h$ th data symbol of the $l$ th interferer, $\tau_{l}$ is its delay, $1 / T_{l}$ its sample rate. Further, $g_{l}(t)$ is the convolution of the $l$ th TD propagation channel response, the TD response of the MCCDMA receiver filter $p_{0}(-t)$ and $p_{l}\left(t-\tau_{l}\right) \exp \left(j 2 \pi f_{c, l} t\right)$, where $p_{l}(t)$ is the transmit pulse of the $l$ th interferer. It is assumed that the interfering symbols are uncorrelated with each other, i.e. $\mathrm{E}\left[z_{h, l} z_{h^{\prime}, l^{\prime}}^{*}\right]=E_{l}^{\prime} \delta_{l l^{\prime}} \delta_{h h^{\prime}}$, where $E_{l}^{\prime}$ is the energy per symbol of the lth interferer, and $\delta_{x x^{\prime}}$ is equal to one if $x=x^{\prime}$ and is equal to zero elsewhere. Further, the interfering data symbols are statistically independent of the MC data symbols. The signal to interference ratio $(S I R)$ at the input of the receiver is defined as [5]

$$
S I R=\frac{2 \sigma_{s}^{2} / T_{0}}{\sum_{l=1}^{N_{I}} \frac{E_{l}^{\prime}}{T_{l}}}
$$

where $\sigma_{s}^{2}=\mathrm{E}\left[\left|s_{j, k}^{(k)}\right|^{2}\right]$ is the average power of the MC transmitted sample per real dimension.

\section{SyNCHRONIZATION AND CHANNEL ESTIMATION}

In [1], the delays $\Delta^{(k)}$ and the channel taps $\mathbf{h}^{(k)}$ for $k=1, \cdots K_{u}$ are estimated without NBI. In this section, we modify the estimation algorithm to estimate $\Delta^{(k)}$ and $\mathbf{h}^{(k)}$ in the presence of an additional NBI. The conceptual block diagram of the receiver is shown in Fig. 2. The receiver of user $k$ employs the MAP (maximum a posteriori probability) algorithm to obtain initial estimates (iteration 1) $\left(\hat{\Delta}^{(k)}(1), \hat{\mathbf{h}}^{(k)}(1)\right)$ for the timing offset $\Delta^{(k)}$ and the channel taps $\mathbf{h}^{(k)}$ (e.g., by exploiting the $N_{p}$ pilot MC symbols). Then, the a posteriori expectations of the transmitted symbols are computed. Although the a posteriori expectations of the symbols serve for decoding and data detection, they can also be used to iteratively improve the timing synchronization and channel estimation. We start again from our observation vector $\mathbf{r}$ (2). Note that the length of this vector (say, $N_{o b s}$ ) is independent of $\Delta^{(k)}$. We can express the observation $\mathbf{r}$ as follows

$$
\mathbf{r}=\sum_{k=0}^{K_{u}-1} \mathbf{S}_{\Delta^{(k)}}^{(k)} \mathbf{h}^{(k)}+\mathbf{w}+\mathbf{I}
$$

where the vector $\mathbf{w}$ is a zero-mean complex Gaussian random variable with variance $\sigma^{2}$ per real dimension. The vector $\mathbf{I}$ corresponds to the NBI signal, and can be represented as

$$
I=\left[r_{I}(0), r_{I}\left(T_{o}\right), \cdots, r_{I}\left(\left(N_{o b s}-1\right) T_{o}\right)\right]
$$

where $r_{I}\left(n T_{o}\right)$ can be obtained by replacing in (3) $t$ by $n T_{o}$. The $N_{o b s} \times L$ matrix $\mathbf{S}_{\Delta^{(k)}}^{(k)}$ is structured as follows:

$$
\mathbf{S}_{\Delta(k)}^{(k)}=\left[\begin{array}{c}
\mathbf{0}_{\Delta^{(k)} \times L} \\
\mathbf{S}^{(k)} \\
\mathbf{0}_{\left(\Delta_{\max }-\Delta^{(k)}+L-1\right) \times L}
\end{array}\right]
$$

where $\mathbf{S}^{(k)}$ is a Toeplitz matrix of the pilot and data symbols of the $k$ th user, and $\mathbf{0}_{L_{1} \times L_{2}}$ is an $L_{1} \times L_{2}$ matrix consisting of all zeros.

\section{A. MAP Estimation}

Our goal is to find the maximum a posteriori (MAP) estimate of the delays and channel impulse responses (CIR) of all the users in the presence of NBI. Introducing a vector $\boldsymbol{\theta}$ containing all these parameters, the MAP estimate is given by

$$
\begin{aligned}
\hat{\boldsymbol{\theta}} & =\arg \max _{\boldsymbol{\theta}} \mathrm{P}(\boldsymbol{\theta} \mid \mathbf{r}) \\
& =\arg \max _{\boldsymbol{\theta}}\left\{\mathrm{P}(\boldsymbol{\theta}) \int \mathrm{P}(\mathbf{r} \mid \boldsymbol{\theta}, \mathbf{s}) \mathrm{P}(\mathbf{s}) d \mathbf{s}\right\}
\end{aligned}
$$

where $\mathbf{s}$ represents the concatenation of all the data symbols of all the users. For the distribution $\mathrm{P}(\mathbf{r} \mid \boldsymbol{\theta}, \mathbf{s})$, we use the Gaussian approximation, which is a reasonable assumption as each component of the vector I consists of the sum of a large number of contributions, such that, according to the central limit theorem, each component of $\mathbf{I}$ has approximate Gaussian distribution. As a consequence, the likelihood function $\mathrm{P}(\mathbf{r} \mid \boldsymbol{\theta}, \mathbf{s})$ may be written as

$$
\begin{aligned}
\mathrm{P}(\mathbf{r} \mid \boldsymbol{\theta}, \mathbf{s}) \propto & \exp \left(-\left(\mathbf{r}-\sum_{k=0}^{K_{u}-1} \mathbf{S}_{\Delta^{(k)}}^{(k)} \mathbf{h}^{(k)}\right)^{H} \mathbf{C}^{-1} \times\right. \\
& \left.\left(\mathbf{r}-\sum_{k=0}^{K_{u}-1} \mathbf{S}_{\Delta^{(k)}}^{(k)} \mathbf{h}^{(k)}\right)\right)
\end{aligned}
$$

where $\mathbf{C}$ is the covariance matrix given by

$$
\begin{aligned}
{[C]_{k, k^{\prime}}=} & \mathrm{E}\left[(\mathbf{w}(k)+\mathbf{I}(k))\left(\mathbf{w}\left(k^{\prime}\right)+\mathbf{I}\left(k^{\prime}\right)\right)^{H}\right] \\
= & 2 \sigma^{2} \delta_{k k^{\prime}}+ \\
& \sum_{l=1}^{N_{I}} E_{l} \sum_{h=-\infty}^{\infty} g_{l}\left(k T_{0}-h T_{l}\right) g_{l}^{*}\left(k^{\prime} T_{0}-h T_{l}\right) .
\end{aligned}
$$

Finding the MAP estimate is intractable in this case since (a) we need to average over all possible transmitted sequences, and (b), the maximization is over a large-dimensional parameter. To solve this problem, we apply the space alternating generalized expectation maximization (SAGE) algorithm [1], [6]. 


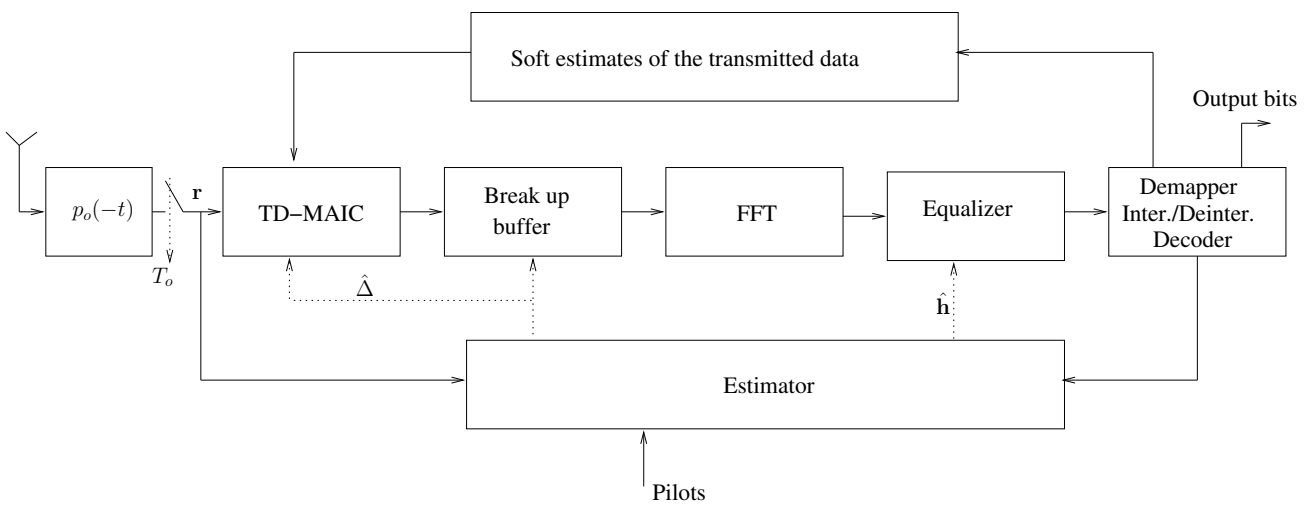

Fig. 2. Detector and estimator for the $k$ th user.

\section{B. Soft Decision-Directed Synchronization and Channel Es- timation}

To estimate the parameter sets $\boldsymbol{\theta}^{(k)} \leftrightarrow\left[\Delta^{(k)}, \mathbf{h}^{(k)}\right]$ for all users, first the base station sorts the received users signals from strongest to weakest power. Then, the parameter sets $\boldsymbol{\theta}^{(k)}$ are estimated sequentially starting with the strongest user. We assume that the channel taps have a Gaussian distribution with known covariance matrix, so that $\mathrm{P}\left(\mathbf{h}^{(k)}\right) \propto$ $\exp \left(-\left(\mathbf{h}^{(k)}\right)^{H} \boldsymbol{\Sigma}_{k}^{-1} \mathbf{h}^{(k)}\right)$, where $\boldsymbol{\Sigma}_{k}$ is the channel covariance matrix associated with the $k$ th user. The delay $\Delta^{(k)}$ is uniformly distributed over its respective domain. A conventional DA algorithm is used to get an initial estimate $\boldsymbol{\theta}^{(k)}(1)$, for all $k=0, \ldots, K_{u}-1$. We follow the same reasoning as in [1] and take into account the statistical properties of NBI through C. The SAGE algorithm then becomes at the $\xi$ th iteration (see Fig. 2):

1) Select the user $k$ for which the delay and CIR estimate must be updated.

2) E-step: The $Q$ function for the SAGE algorithm may be written as:

$$
\begin{aligned}
Q\left(\boldsymbol{\theta}^{(k)} \mid \widehat{\boldsymbol{\theta}}^{(k)}(\xi-1)\right)= \\
\quad \mathrm{E}_{\mathbf{s}}\left[\log \mathrm{P}\left(\widetilde{\mathbf{x}}^{(k)} \mid \boldsymbol{\theta}^{(k)}\right)\right]+\log \mathrm{P}\left(\boldsymbol{\theta}^{(k)}\right) \propto \\
\quad 2 \operatorname{Re}\left\{\left(\widetilde{\mathbf{x}}^{(k)}\right)^{H} \mathbf{C}^{-1} \widetilde{\mathbf{S}}_{\Delta(k)}^{(k)} \mathbf{h}^{(k)}\right\}-\left(\mathbf{h}^{(k)}\right) \boldsymbol{\Sigma}_{k}^{-1} \mathbf{h}^{(k)} \\
\quad-\left(\mathbf{h}^{(k)}\right)^{H} \widetilde{\mathbf{S}}_{\Delta^{(k)}}^{(k) H} \mathbf{C}^{-1} \widetilde{\mathbf{S}}_{\Delta^{(k)}}^{(k)} \mathbf{h}^{(k)}
\end{aligned}
$$

where $\widetilde{\mathbf{x}}^{(k)}$ is the received signal corresponding to the user $k$ which is obtained by removing the contributions of all users except user $k$ from the total received signal $\mathbf{r}$, and $\widetilde{\mathbf{S}}_{\Delta^{(k)}}^{(k)}$ is obtained by replacing each entry in $\mathbf{S}_{\Delta^{(k)}}^{(k)}$ with the corresponding a posteriori expectation.

3) M-step: now we update the estimate of $\boldsymbol{\theta}^{(k)}$ :

$$
\boldsymbol{\theta}^{(k)}(\xi)=\arg \max _{\boldsymbol{\theta}^{(k)}}\left\{Q\left(\boldsymbol{\theta}^{(k)} \mid \widehat{\boldsymbol{\theta}}^{(k)}(\xi-1)\right)\right\}
$$

4) The previous steps are repeated for all users in the $\xi$ th iteration.

For each value of $\Delta$, the corresponding estimated channel $\hat{\mathbf{h}}_{\Delta}^{(k)}(\xi)$ can be found in closed form by maximizing (12) as:

$$
\hat{\mathbf{h}}_{\Delta^{(k)}}^{(k)}(\xi)=\left(\Sigma_{k}^{-1}+\widetilde{\mathbf{S}}_{\Delta^{(k)}}^{H} \mathbf{C}^{-1} \widetilde{\mathbf{S}}_{\Delta^{(k)}}\right)^{-1} \widetilde{\mathbf{S}}_{\Delta^{(k)}}^{H} \mathbf{C}^{-1} \mathbf{r} .
$$

Accordingly, the estimated delay $\hat{\Delta}^{(k)}(\xi)$ can be obtained by using a one dimensional linear search:

$$
\begin{aligned}
\hat{\Delta}^{(k)}(\xi)=\arg \max _{\Delta^{(k)}} & \left\{2 \operatorname{Re}\left\{\left(\widetilde{\mathbf{x}}^{(k)}\right)^{H} \mathbf{C}^{-1} \widetilde{\mathbf{S}}_{\Delta^{(k)}}^{(k)} \mathbf{h}_{\Delta^{(k)}}^{(k)}\right\}\right. \\
& -\left(\mathbf{h}_{\Delta^{(k)}}^{(k)}\right)^{H} \boldsymbol{\Sigma}_{k}^{-1} \mathbf{h}_{\Delta^{(k)}}^{(k)} \\
- & \left.\left(\mathbf{h}_{\Delta^{(k)}}^{(k)}\right)^{H} \widetilde{\mathbf{S}}_{\Delta^{(k)}}^{(k) H} \mathbf{C}^{-1} \widetilde{\mathbf{S}}_{\Delta^{(k)}}^{(k)} \mathbf{h}_{\Delta^{(k)}}^{(k)}\right\} .
\end{aligned}
$$

Finally, the estimated channel taps $\hat{\mathbf{h}}^{(k)}(\xi)=\hat{\mathbf{h}}_{\hat{\Delta}^{(k)}(\xi)}^{(k)}(\xi)$ can be obtained by replacing in (14) $\Delta^{(k)}$ by $\hat{\Delta}^{(k)}(\xi)$.

\section{NUMERICAL RESULTS}

To validate the proposed algorithms, we have carried out Monte Carlo simulations. We considered a system with $K_{u}=$ 5 users, using a convolutional code with constraint length 5 , rate $R=1 / 2$ and polynomial generators $(23)_{8}$ and $(35)_{8}$. A block length of $N_{b}=528$ information bits was chosen, leading to $N_{c}=1056$ coded bits. Coded bits are Gray-mapped onto an 8-PSK constellation resulting in $N_{d}=352$ data symbols to which $N_{p}=328$ 8-PSK pilots symbols are appended for initial channel parameter estimation. This sequence of $N_{d}+N_{p}=$ 384 8-PSK symbols is broken up into $M=12$ blocks of $P=$ 32 8-PSK symbols per block. Spreading sequences are realvalued Walsh-Hadamard sequences, with chips belonging to $\left\{-\frac{1}{\sqrt{N_{s}}},+\frac{1}{\sqrt{N_{s}}}\right\}$ and have a length $N_{s}=16$, leading to $N=$ $P N_{s}=512$ sub-carriers. The channel has length $L=15$ and is modeled with independent components, each being a zeromean complex Gaussian random variable with an exponential power delay profile:

$$
\mathrm{E}\left[\left(h^{(k)}(l)\right)^{*} h^{\left(k^{\prime}\right)}\left(l^{\prime}\right)\right]=\delta_{k-k^{\prime}} \delta_{l-l^{\prime}} \sigma_{h^{(k)}}^{2} \exp (-l / 5)
$$

where $l=0, \ldots, L-1$ and $\sigma_{h^{(k)}}^{2}$ is chosen such that the average energy per sub-carrier is normalized to unity. Hence, the energy of the channel is concentrated mainly in the first few channel taps. The reference propagation delay is $\Delta=$ 17. The timing shifts $\Delta^{(k)}$ are fixed to $7,11,25,37,17$ for $k=1, \ldots, 5$, respectively. A cyclic prefix of length $\nu=16$ is employed.

To generate the NBI signal, we assume that the transmit filters are square-root raised-cosine filters with roll off factors 


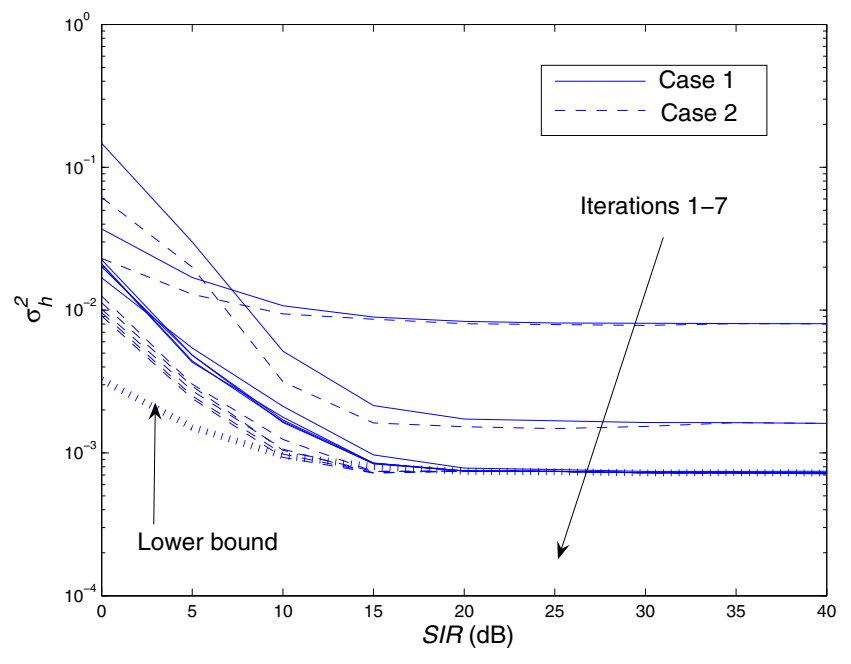

Fig. 3. Variance of the estimated CIRs for synchronous users for different iterations, $E_{b} / N_{o}=12 \mathrm{~dB}$.

$\alpha_{0}=0.25$ and $\alpha_{l}=0.5$ for $\mathrm{MC}$ and interfering signals, respectively. We assume $N_{I}=1$ and $f_{c, 1}=0$. The bandwidth of the MC and interferer spectrum equal $B_{0}=\frac{1}{T_{0}}=1024$ $\mathrm{kHz}$ and $B_{1}=\frac{1}{T_{1}}=25 \mathrm{kHz}$ respectively. We use QPSK modulation for the interferer signal. The time delay of the interferer equals $\tau_{1}=0$.

The performance will be evaluated in terms of mean squared estimation error (MSE) and bit error rate (BER). Where applicable, the BER of the code-aided estimator (referred to as the CA estimator) is compared to a system with full knowledge of the channel and synchronization parameters, while the MSE is compared to an estimator which has perfect knowledge of all the data symbols (referred to as the full-DA estimator).

To evaluate the performance of the proposed algorithm, we consider two cases. In the first case, we do not take into our account the statistics of the NBI in the estimation process. Accordingly, the covariance matrix $\mathbf{C}$ is a diagonal one, where each diagonal element equals the variance of AWGN plus MAI $\left(\sigma^{2}\right)$. This case represents the effects of the NBI on the iterative algorithm proposed in [1]. In the second case, we consider the NBI statistics in the estimation process.

Fig. 3 shows the mean square estimation error $\sigma_{h}^{2}$ as a function of $S I R$ at $E_{b} / N_{0}=12 \mathrm{~dB}$ for the two cases, assuming perfect timing synchronization. As can be observed, a strong improvement of $\sigma_{h}^{2}$ is achieved after 3 iterations for the two cases. At low values of $S I R, \sigma_{h}^{2}$ decreases for increasing $S I R$. At high values of $S I R, \sigma_{h}^{2}$ is independent of the $S I R$ as the effect of the NBI diminishes. Further, the MSE performance of case 2 is slightly better than the MSE performance of case 1 at low values of SIR. However, at high values of $S I R, \sigma_{h}^{2}$ for both algorithms converges to the same asymptote, corresponding to the case where no interference is present. This asymptote is determined by the signal to noise ratio $\left(E_{b} / N_{0}\right)$. Moreover, we notice that at low $S I R$ values, $\sigma_{h}^{2}$ at the second iteration for the two cases is worse than for the first iteration. This can be explained as follows. In the second iteration (i.e. the first iteration where the data symbols are used in the estimation), the reliability of the data symbols is low at low $S I R$ because they are affected by

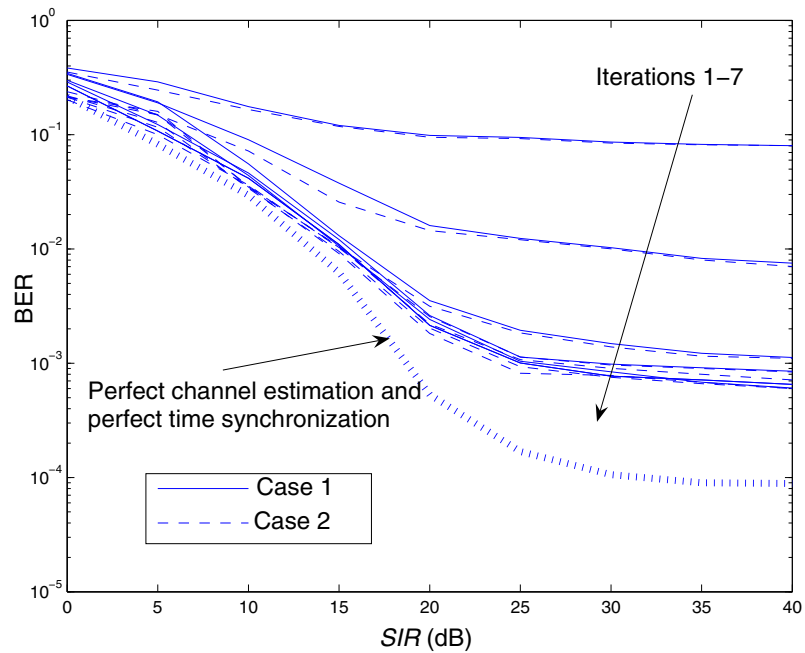

Fig. 4. BER for 5 synchronous users, $E_{b} / N_{o}=12 \mathrm{~dB}$.

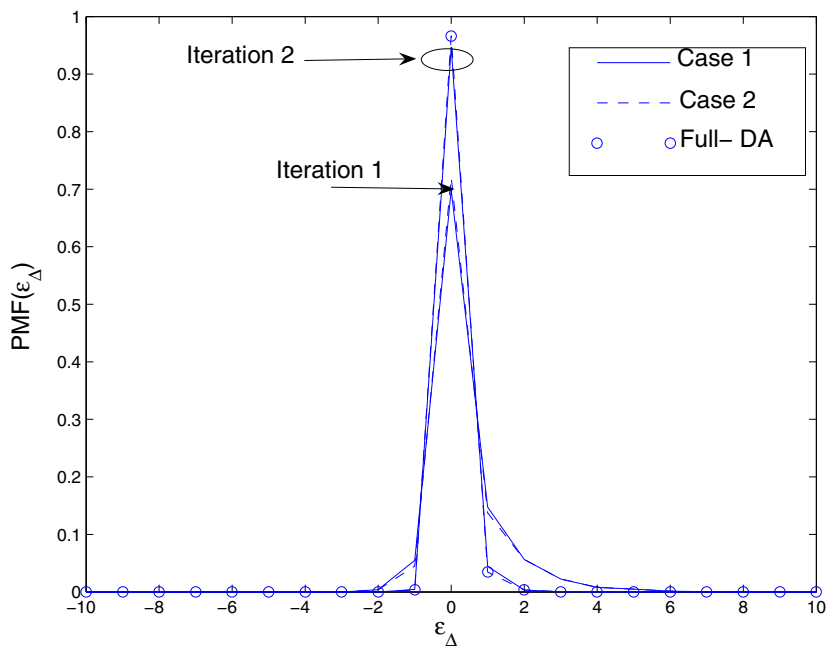

Fig. 5. PMF of timing estimation error at $E_{b} / N_{o}=12 \mathrm{~dB}, S I R=$ $15 \mathrm{~dB}$ and $K_{u}=5$ users for two new users accessing the system (two users are asynchronous and three users are synchronous).

strong interference. However, when the number of iterations increases, the reliability of the data detection and estimation improves, as more information is used than in the DA only case (i.e. the first iteration).

Fig. 4 shows the corresponding BER performance. The BER of perfect channel estimation and perfect synchronization is also shown. As can be observed, the soft information aided estimator is able to improve the BER performance after 3 iterations for the two cases. Further, we notice that case 2 gives a slight improvement as compared to case 1 .

As the MSE of the delay $\Delta^{(k)}$ does not give a clear picture, the average probability mass function (PMF) of $\epsilon_{\Delta}$ is considered (see Figs 5 and 6) for the two cases, $K_{u}=5$ users and $E_{b} / N_{o}=12 \mathrm{~dB}$ when two and five users are asynchronous respectively. From the figure, it follows that the SAGE-based frame synchronizer will produce estimates that deviate only slightly from the true value, i.e. the estimation error $\epsilon_{\Delta}$ is in 


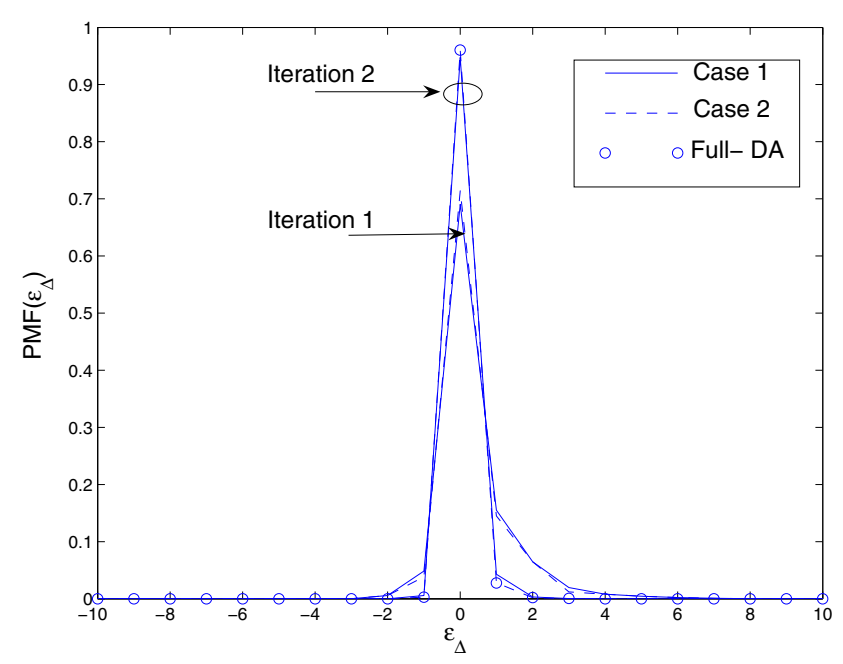

Fig. 6. PMF of timing estimation error at $E_{b} / N_{o}=12 \mathrm{~dB}, S I R=$ $15 \mathrm{~dB}$ and $K_{u}=5$ users for five new users accessing the system (five users are asynchronous).

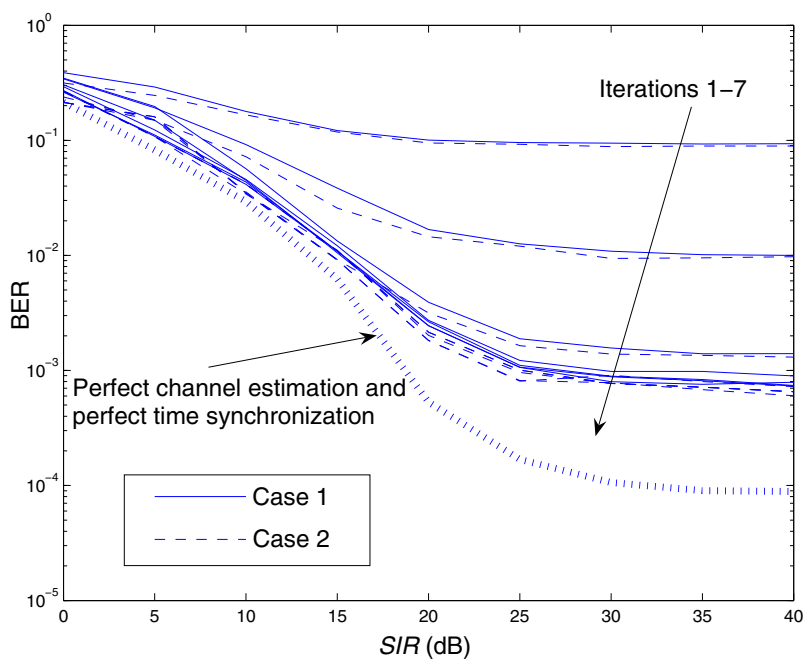

Fig. 7. BER for two new users accessing the system, $E_{b} / N_{o}=12$ $\mathrm{dB}$ and $K_{u}=5$ users (two users are asynchronous and three users are synchronous).

the range $[-1,2]$ samples after two iterations for the two cases. Moreover, it can be noticed that the CA estimator (iteration 2) yields a PMF close to the full-DA estimator. Further, we notice that case 2 gives a slight improvement as compared to case 1. This can be explained as follows: the covariance matrix element $[\mathbf{C}]_{k, k^{\prime}}$ represents the sum of the product of the interferer pulse after matched filtering with its delayed version over an integer number of sample intervals $T_{0}$, see (11). If the interferer has a small bandwidth as compared to the MC signal, it turns out that the significant elements of $\mathbf{C}$ occur at the main diagonal and a few diagonals around it. The further away the elements from the main diagonal, the smaller the elements will be. Due to this property of the covariance matrix, we do not get a significant estimation improvement by taking the statistics of the NBI into account.

Figs. 7 and 8 report the corresponding BER performance

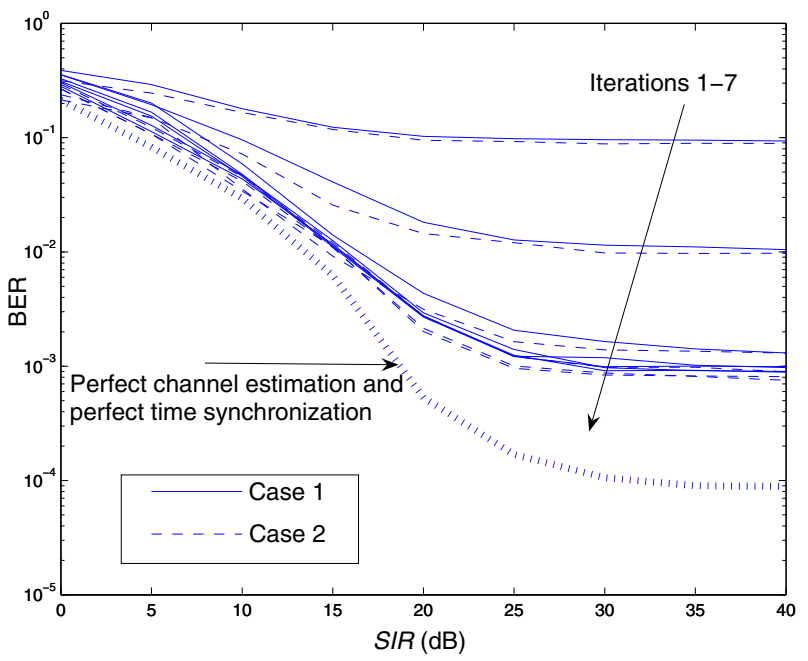

Fig. 8. BER for five new users accessing the system, $E_{b} / N_{o}=12$ $\mathrm{dB}$ and $K_{u}=5$ (five users are asynchronous).

for joint synchronization and channel estimation for the two cases for $K_{u}=5$ when two and five users are asynchronous respectively. As expected, the data aided estimator (iteration 1) gives rise to large degradations. On the other hand, the SAGE algorithm has good performance after 3 iterations. Further, the BER of case 2 shows a gain of approximately $0.5-1 \mathrm{~dB}$ as compared to case 1 .

\section{CONCLUSiOnS}

We investigated the effect of NBI on the code aided MAP joint frame synchronization and channel estimation algorithm for an uplink MC-CDMA system. Further, we modified the algorithm by taking the statistics of the NBI into account. The results indicate that the modified algorithm has better mean square channel estimation error than the original algorithm. The probability mass functions of timing estimation error for the two algorithms almost coincide with the full data aided case. Further, the BER of the modified algorithm slightly outperforms that of the original algorithm.

\section{REFERENCES}

[1] M. Guenach, M. Marey, H. Wymeersch, H. Steendam, and M. Moeneclaey. "Turbo Estimation and Equalization for Asynchronous Uplink MC-CDMA ". IEEE Transactions on Wireless Communications, vol. 7(4):1217-1226, Apr. 2008.

[2] L. Hanzo, M. Munster, B. Choi, and T. Keller. "OFDM and $M C$ CDMA for Broadband and Multi-User Communications, WLANs and Broadcasting". Wiley, 2004.

[3] S. Hara and R. Prasad. "Overview of Multi-Carrier CDMA". IEEE Communications Magazine, vol. 35(12):pp. 126-132, Dec. 1997.

[4] http://www.b-vhf.org.

[5] M. Marey and H. Steendam. "Analysis of the Narrow Band Interference Effect on OFDM Timing Synchronization". IEEE Transactions on Signal Processing, vol. 55(9):pp. 4558-4566, Sep. 2007.

[6] J. A. Fessler and A. O. Hero. "Space Alternating Generalized Expectation Maximization Algorithm". IEEE Transactions on Signal Processing, vol. 42(10):pp. 2664-2677, Oct. 1994. 